\title{
¿Es Siempre Exitosa la Inclusión Educativa? Resultados Comparativos del Sistema Regular y Especial
}

\section{Is Educational Inclusion Always Successful? Comparative Results of the Regular and Special System}

\author{
Ricardo Rosas* \\ Victoria Espinoza \\ Elisa Hohlberg \\ Sanndy Infante \\ Pontificia Universidad Católica de Chile, Chile
}

\begin{abstract}
La implementación de sistemas de educación inclusiva supone un desafío tanto a nivel de escuelas como respecto del desarrollo de las políticas públicas que la sustenten. La presente investigación compara los resultados obtenidos en habilidades cognitivas, precursores de la lectura, matemática inicial y problemas emocionales en estudiantes de educación inicial, 29 de ellos con discapacidad visual y 22 con discapacidad auditiva. Los resultados se analizaron en función del tipo de sistema escolar al que pertenecían los estudiantes, es decir, si estaban integrados en escuela regulares o si estudiaban en escuelas especiales. Los resultados indican la presencia de diferencias en el desempeño en las diversas áreas evaluadas según el tipo de discapacidad y el sistema escolar. Los resultados se discuten en torno a la importancia de diseñar estrategias y políticas educativas que se adapten a las características y necesidades de los estudiantes, considerando los recursos, tanto materiales como humanos, necesarios para generar un sistema educativo realmente inclusivo.
\end{abstract}

Descriptores: Resultados educativos; Educación especial; Educación inclusiva; Discapacidad auditiva; Discapacidad visual.

The implementation of inclusive education systems is a challenge both at the school level and with respect to the development of public policies that support it. The present research compared the results obtained in cognitive skills, reading precursors, initial mathematics and emotional problems in initial education students, 29 of them with visual disabilities and 22 with hearing disabilities. The results were analyzed according to the type of school system to which the students belonged, that is, if they were integrated in regular schools or if they studied in special schools. The results indicate the presence of differences in performance in the various areas evaluated according to the type of disability and the school system. The results are discussed around the importance of designing educational strategies and policies that adapt to the characteristics and needs of the students, considering the resources, both material and human, necessary to generate a truly inclusive educational system.

Key words: Educational outcomes; Special education; Inclusive education; Hearing impairment; Visual impairment. 


\section{Introducción}

Desde que en 1978 se hiciera público el célebre informe Warnock, comenzó un proceso progresivo de cambio en la forma como se consideraba el rol de la educación especial y la necesidad de avanzar en políticas públicas, para pasar de una educación segregada a una inclusiva. Un impulso definitivo a este propósito lo permitió la Declaración de Salamanca, el año 1994. Esto significó en algunos casos la disolución de sistemas de educación especial, aunque en muchos países, incluido Chile, ha prevalecido un sistema mixto. De hecho, aunque en Chile entre 2010 y 2020 ha habido cierres de escuelas especiales, particularmente de sordos, si se revisa la matrícula general de escuelas especiales disponible en la página web del Ministerio de Educación se podrá observar que el número de matriculados en la educación especial no sólo no ha mostrado una disminución, sino incluso un leve aumento (de 140.000 en 2010 a 180.000 en 2020) (Ministerio de Educación, 2010, 2020).

La educación especial proveyó, de forma histórica, de oportunidades de aprendizaje a estudiantes que estaban normalmente fuera del sistema escolar, pero a nivel global la tendencia ha sido migrar de la educación especial a la educación inclusiva (Biermann y Powell, 2016). Muchos países han asumido el desafío de desarrollar sistemas educativos progresivamente inclusivos (ver por ejemplo, Richardson y Powell, 2013; para un análisis global, Biermann y Powell, 2014, que comparan Islandia, Noruega y Alemania, Rosas et al., 2019, comparando Chile, España y Finlandia).

Las políticas educativas en pro de una mayor inclusión han estado fundamentadas, sin embargo, en principios valóricos más que en evidencia científica. Como plantea Hornby (2014), en su libro Educación Especial Inclusiva, parece haber una confusión en la base de investigación necesaria para fundamentar una educación inclusiva. En efecto, defensores de la inclusión a ultranza, piensan que la investigación que justifique la inclusión es innecesaria, o ya existe. Tanto la escasa investigación reportada a la fecha, como la evidencia que existía en los tiempos de la publicación del mencionado libro, parecen apoyar el aserto que en este ámbito se ha dado por supuesto que esta investigación es más bien innecesaria (Hornby, 2014).

Una revisión de Lindsay (2007) reporta que la evidencia a favor de las políticas inclusivas no es concluyente, sugiriendo que se debiera establecer una adecuada base de investigación para apoyar estas políticas. Este hallazgo es apoyado por revisiones ulteriores, como la de Kauffman y Hallahan (2011) o la de Farrell, (2010), quien cita una serie de artículos de estudios relevantes, muchos de los cuales reportan resultados negativos respecto al impacto de la educación inclusiva. Ahora bien, existen algunos metaanálisis (Hehir et al., 2016; Pocock y Miyahara, 2017) que refieren a los beneficios de la inclusión educativa, entendiéndose que no solo implica a estudiantes con necesidades educativas especiales. No obstante lo anterior, lo que resulta claro de la revisión de la literatura, es que falta aún evidencia empírica de los resultados comparados de largo plazo de niños con necesidades educativas especiales (NEE) tanto en aulas inclusivas como en aulas de educación especial.

El presente estudio tiene por objetivo, entonces, aportar alguna evidencia en torno a cuán exitosa es la implementación de programas de inclusión educativa en nuestro país. Porque si bien compartimos plenamente los principios de la plena inclusión, también pensamos que la investigación en este ámbito no solo es necesaria, sino imprescindible. Porque solo con datos de calidad, es posible señalar si las políticas inclusivas están siendo bien implementadas, o tienen aspectos por mejorar. Para efectos de este estudio, nos 
centraremos estudiar los rendimientos cognitivos y de desarrollo socioafectivo en dos poblaciones de estudiantes en dos modalidades de escolaridad: estudiantes con Discapacidad Auditiva (DA) y estudiantes con Discapacidad Visual (DV), que asisten a Escuelas Especiales o Escuelas Regulares.

\section{Antecedentes teóricos}

A nivel nacional, la inclusión de los niños y niñas con Necesidades Educativas Especiales (NEE) en escuelas regulares se gestiona por medio de los Programa de Integración Escolar (PIE). Mediante estos el Estado entrega recursos humanos y materiales adicionales al colegio, que le permitan brindar al niño o niña los apoyos que requiera, según la NEE que presente (Ministerio de Educación). Asimismo, existen escuelas especiales, las cuales son centros especializados que enseñan a alumnos y alumnas, que presentan alguna discapacidad o necesidad educativa especial, según sus requerimientos específicos (Ministerio de Educación). El tipo de educación al que el alumno acceda (escuela especial o escuela regular a través del PIE), vendrá determinada a través de una decisión informada que debe tomar la familia y/o el propio estudiante cuando esto se le haga posible (Ministerio de Educación).

En Chile no hay a la fecha, investigación empírica acerca de los efectos de la educación inclusiva versus la especial. Sin embargo, un estudio reciente abordó el tema de los efectos de la educación inclusiva sobre el rendimiento de los estudiantes incluidos y sus pares. Contreras, Brante, Espinoza y Zuñiga (2020) estimaron el efecto que tiene la educación en un aula con al menos un alumno con NEE, sobre el rendimiento académico de los compañeros y compañeras sin NEE, antes y después de la política de inclusión implementada en el 2010. Esta reforma incluyó: (1) una definición más amplia de las NEE, que lleva al reconocimiento y educación de los estudiantes con dichas necesidades, así como a la entrega de recursos adicionales para esto; (2) el establecimiento de protocolos más estrictos y específicos para el tratamiento y educación de estudiantes con necesidades educativas permanentes (NEEP) y la suma de otros nuevos para los alumnos con necesidades educativas transitorias (NEET)2. Para estimar dicho efecto de manera fija, los

\footnotetext{
${ }^{1}$ Los estudiantes con NEEP reciben 286 dólares al mes antes y después de la reforma. Este total comprende el subsidio regular (US \$ 94, el monto básico que reciben todos los estudiantes) y un subsidio especial (US \$ 192). Los estudiantes con NEET, que no fueron reconocidos como tales, recibieron el subsidio mensual regular (US \$ 94) antes de la reforma. Luego de la implementación de la nueva política, a los estudiantes con NEET se les otorgó un subsidio adicional de US \$ 155 , que incrementó su subsidio mensual a US \$ 249.

2 Antes de 2010, la ley no definía requisitos específicos para el tratamiento de estudiantes con necesidades educativas especiales específicas en el aula. La principal característica del programa de integración utilizado antes del cambio de política era la "sala de recursos", donde los estudiantes con NEE recibían formación separada del resto de la escuela, recibiendo apoyo y atención. Dependiendo de su diagnóstico, los estudiantes podían (1) asistir a un salón de clases regular, (2) asistir tanto a un salón de clases regular como al salón de recursos, o (3) asistir sólo al salón de recursos. En el último caso, estos podían unirse a sus compañeros en el aula regular, para eventos especiales o actividades recreativas.

Después de 2010, la ley proporcionó instrucciones específicas para abordar cada una de las NEE reconocidas. Es decir, un estudiante con NEE que asiste a una escuela con PIE, sólo puede recibir clases en un salón de clases regular, debido a la eliminación del aula de recursos. La ley también brindó información más específica sobre los componentes mínimos de la asistencia para cada NEE identificada.
} 
investigadores utilizaron datos longitudinales del año 2007 (antes de la reforma del 2010) y del 2011 (después de la reforma del 2010) de una cohorte de estudiantes, recolectados a través de dos fuentes de información: (1) bases de datos del Ministerio de Educación, a través de las cuales se identificaron a los estudiantes con NEE y (2) bases de datos del SIMCE, que permitieron observar los puntajes individuales de los estudiantes en pruebas estandarizadas y algunas de sus características observables. Para construir un panel del desempeño académico de los estudiantes, antes y después de la reforma, se usaron los puntajes de matemática y lenguaje del SIMCE 2007 (cuarto básico) y del SIMCE 2011 (octavo básico) $^{3}$. Los resultados mostraron que la inclusión de alumnos con NEEP y NEET tuvo un efecto negativo bajo en el desempeño académico de sus compañeros y compañeras sin NEE antes de la reforma del 2010, esto quiere decir que, antes de la reforma del 2010, la inclusión de alumnos con NEE sí afectó el desempeño de sus compañeros, pero el efecto fue bajo. Cuando el cambio en la política pública comienza a operar, el efecto negativo de la integración de alumnos y alumnas con NEET y NEEP en los puntajes de las pruebas estandarizadas se neutraliza por completo. Por consiguiente, los autores concluyen que el mayor reconocimiento y administración de recursos para las NEE, establecidas en la reforma del 2010, podrían estar explicando que la integración de estudiantes con NEE ya no estuviera afectando los resultados académicos obtenidos por sus compañeros y compañeras sin NEE.

La matrícula de escuelas especiales entre los años señalados tuvo un leve aumento en el caso de la DV (de 389 a 420) y una fuerte disminución en el caso de la DA (de 734 a 345). La implementación de la nueva política de inclusión vigente desde el año 2010, ha abierto muchas interrogantes respecto de la calidad de la educación recibida, particularmente entre el sistema de educación especial, y los estudiantes incluidos en aulas regulares. Dado que los estudiantes con DV y DA han sido atendidos históricamente en escuelas especiales, por profesionales entrenados en didácticas específicas para abordar las particularidades de la alfabetización inicial de estas dos poblaciones, resulta especialmente importante tener datos respecto de los resultados que obtienen en aulas inclusivas versus en aulas segregadas. A continuación, haremos una somera revisión del estado del arte internacional de la inclusión de estudiantes con discapacidad, con un énfasis especial en estudiantes con DV y DA.

\subsection{Discapacidad auditiva}

La DA es probablemente la condición de discapacidad más heterogénea, con muchas variables a tomar en cuenta a la hora de describirla: etiología, edad al diagnóstico, desarrollo, habilidad cognitiva, nivel socioeconómico, apoyo parental, tipo de comunicación preferente (oral o lengua de señas), historia educacional, etc. Asimismo, el desarrollo de tecnologías como los implantes cocleares ha agregado aún más variables: edad al momento del implante, tipo de implante, nivel de pérdida auditiva antes y después de la implantación o uso de audífono, estrategia de procesamiento y manejo del dispositivo (Archbold, 2015). Esto conlleva, entre otras cosas, a que exista una importante diversidad

${ }^{3}$ Las escuelas privadas fueron excluidas del estudio ya que representan sólo a una pequeña parte del número total de estudiantes en Chile y trabajan con un número pequeño de estudiantes. Asimismo, en los resultados del SIMCE, ya que los niños con NEE tienen permitido saltarse la prueba y sus puntajes no son considerados en los logros educativos, en este estudio no fue posible analizar los resultados considerando los puntajes obtenidos en el SIMCE por estudiantes con NEE. 
en cuanto a las modalidades de comunicación existentes dentro de este mismo grupo (Crowe, 2018).

El tamizaje temprano y la intervención adecuada a través de tecnologías auditivas han permitido en varios casos, que los niños y niñas con DA adquieran un desarrollo posterior del habla, lenguaje y desempeño funcional (Ching, Dillon, Leigh, y Cupples, 2018; Cupples et al., 2018; Organización Mundial de la Salud, 2016). Lo anterior ha llevado, a que los sordos implantados, opten cada vez más por la educación regular (Archbold, Nikolopoulos, Lutman, y O’Donoghue, 2002; de Raeve, Baerts, Colleye, y Croux, 2012; Moog, Geers, Gustus, y Brenner, 2011).

Sin embargo, la progresiva inclusión de sordos implantados en aulas regulares no está exenta de dificultades. En un estudio realizado por Allen, Yen Ng, y Archbold (2016) en donde se realizó una encuesta de satisfacción con respecto al sistema escolar a padres Europeos de niños sordos, estos concluyen que los establecimientos regulares podrían presentar una falta de flexibilidad para el abordaje de las necesidades individuales de cada estudiante con DA. Asimismo, la falsa creencia de que los niños o niñas implantados necesariamente contaban con los mismos niveles audiológicos que sus pares, generaba que estos recibieran menos apoyos de los que realmente necesitaban. Para lo anterior, los investigadores realizaron una encuesta a padres de niños sordos, para estudiar su satisfacción respecto de los servicios educativos recibidos por sus hijos. Se recibieron respuestas de 231 participantes de 20 países. Estos proveyeron información del tipo de pérdida auditiva de sus hijos, de las tecnologías de audición utilizadas, del modo de comunicación, de la provisión educacional y de las ayudas recibidas de especialistas. Un $65 \%$ de los niños tenían una pérdida auditiva profunda, y al menos un $68 \%$ utilizaban al menos un implante coclear. Un $68 \%$ de los niños asistían a escuelas regulares. Un $65 \%$ se comunicaba exclusivamente de forma oral, un $27 \%$ en forma oral y lengua de señas y un $18 \%$ solo en lengua de señas. No se especifican estos porcentajes divididos por el tipo de educación recibida.

Por mínimo que sea el déficit auditivo, la evidencia es contundente en mostrar que estos estudiantes tienden a mostrar problemas no menores de ajuste escolar: problemas atencionales, desarrollo alterado del lenguaje y dificultades para adquirir habilidades lectoras (Goldberg y Richburg, 2004; Moeller et al., 2007). Los estudiantes sordos profundos, por otra parte, muestran consistentemente rendimientos más bajos en pruebas estandarizadas que los estudiantes con DA leve a moderado (Mitchell y Karchmer, 2012).

Respecto al ajuste socioemocional en escuelas regulares, Antia y otros (2011), encontraron que estudiantes sordos educados con oyentes, muestran habilidades sociales en el mismo rango que sus pares oyentes, cuando tienen apoyo profesional especializado, apoyo en comunicación y lenguaje y son incluidos en actividades grupales. Por su parte, Wolters y otros (2014) proponen que no existe un solo tipo de intervención para lograr la aceptación de un niño con DA, sino que, por el contrario, para realizar intervenciones psicosociales en estudiantes con DA es necesario generar estrategias a largo plazo que consideren diversos factores del alumno, tales como el género, perfiles de conducta del alumno, tipo de establecimiento y modo de comunicación predominante.

En esta línea, una pregunta que ha llamado la atención de diferentes investigadores, es el aprendizaje, rendimiento y ajuste socioemocional de estudiantes con DA, dependiendo si asisten a escuelas inclusivas, segregadas, o algún sistema mixto (Knoors y Marschark, 2014). Fellinger y otros (2009), encontraron que los estudiantes con un nivel de habla 
apropiado contaban con bienestar psicológico solo si asistían a escuelas regulares (donde el medio de comunicación predominantes es el lenguaje oral) y presentaban más problemas psicosociales cuando asistían a escuelas especiales (donde el medio de comunicación predominantes es la lengua de señas). Por el contrario, los estudiantes cuyo medio de comunicación era la lengua de señas, tenían mayor bienestar psicológico cuando asisten a escuelas especiales que a escuelas regulares.

Por su parte Hintermair (2013), estudió los problemas conductuales de los estudiantes con DA y su relación con las funciones ejecutivas y las habilidades comunicativas. Para esto evaluó por medio de los profesores, a 214 estudiantes con DA con la versión alemana del Behavior Rating of Executive Function (BRIEF-D), y un cuestionario que mide competencia comunicativa y problemas conductuales (versión alemana del Strenghts and Difficulties Questionnaire, SDQ-D). Los resultados mostraron que, en casi todas las escalas, los estudiantes con DA mostraban una tasa de problemas significativamente mayor en las funciones ejecutivas, en comparación con la muestra normativa de niños y niñas oyentes. En el grupo de alumnos y alumnas con DA, los estudiantes que pertenecían a escuelas regulares mostraron mejores puntajes que los estudiantes que asistían a escuelas para sordos. Asimismo, los análisis de regresión mostraron la importancia que tiene de las funciones ejecutivas y las competencias comunicativas en los problemas conductuales.

Una precaución que plantean a este respecto Stinson y Kluwin (2012), es que juzgar la efectividad de diferentes sistemas educativos para los estudiantes sordos, es "difícil, sino imposible, debido a las diferencias de los niños que asisten a estos programas” (p. 51). Después de revisar la investigación disponible y metaanálisis en el área, estos autores concluyen que el tipo de educación al que asisten explica menos del $5 \%$ de la varianza de los resultados de logro, probablemente debido más a diferencias al ingreso a alguno de los sistemas, que a diferencias atribuibles a las características de las escuelas. Asimismo, estos autores estiman que cerca del $25 \%$ de la varianza del rendimiento de estos estudiantes, se explica por características como la edad, el tipo de discapacidad y las habilidades lingüísticas. En esta línea, los autores explican que varios estudios sugieren que las diferencias "a priori” de los estudiantes, que resultan en la matrícula de estos en distintos sistemas educativos, son el factor más relevante en los logros de los estudiantes con discapacidad auditiva. Y por lo tanto, el sistema educativo por sí mismo no es el factor crítico (Stinson y Kluwin, 2012).

Otros autores sugieren que la variable más importante para explicar la variabilidad del rendimiento es la calidad de los profesores. Incluso hay fuerte evidencia que muestra que estudiantes sordos atendidos por profesores calificados en didácticas específicas para sordos, pueden rendir tan bien como sus pares oyentes (Knoors y Marschark, 2014, Marschark et al., 2008;).

\subsection{Discapacidad visual}

La discapacidad visual, al contrario de la auditiva, no presenta los desafíos didácticos que implica en la práctica, ser educada en una lengua a la cual se tiene un acceso limitado, como es el caso de los estudiantes sordos. Los estudiantes ciegos sólo requieren de didácticas específicas para el dominio de la lectura del código Braille, al inicio de su escolarización, y del dominio de dispositivos tecnológicos de accesibilidad a las TICs (por ejemplo, lectores de pantalla tipo JAWS o NVDA). Por ello, el movimiento inclusivo mundial ha llevado a que los estudiantes ciegos sean progresivamente atendidos en los 
sistemas de educación regular. En el Reino Unido, por ejemplo, aproximadamente el 70\% de los más de 25.000 estudiantes ciegos, son atendidos en el sistema regular de educación, con uno o dos estudiantes ciegos en una clase de hasta 30 pares videntes (Morris y Smith, 2008, citado en Metatla, 2017).

Para efectos del presente estudio, no fue posible encontrar evidencia de resultados de aprendizajes cognitivos diferenciados por lugar de escolarización (escuela regular o escuela especial). Pero el ajuste socioemocional de niños con DV en general, sí es un tema que ha concitado la atención de diferentes grupos de investigación. Dado que los niños con DV tienen una desventaja objetiva para monitorear el entorno, se ha demostrado que la DV afecta la interacción y habilidades sociales de los estudiantes con esta condición (Alabdulkader y Leat, 2010; Celeste y Grum, 2010). Los estudiantes con DV tienen capacidad de reconocer las emociones desde la perspectiva propia del niño y desde el compañero (Pring y Tadic, 2010). No obstante, estudios han demostrado que en tareas que involucran el reconocimiento de estados mentales más complejos, los alumnos con DV son menos eficientes que sus pares de desarrollo típico (Dyck et al., 2004, citado en Pring y Tadic, 2010).

El único estudio al que pudimos acceder, que compara rendimiento entre estudiantes con DV de contextos incluidos vs segregados, fue el de Heyl y Hintermair (2015), quienes evaluaron a 226 niños con DV con la versión alemana del Behavior Rating of Executive Function (BRIEF-D), y un cuestionario que mide competencia comunicativa y problemas conductuales (versión alemana del Strenghts and Difficulties Questionnaire, SDQ-D). Los resultados muestran diferencias altamente significativas en todos los dominios de las funciones ejecutivas: los estudiantes con DV rinden consistentemente más bajo que sus pares videntes de la muestra normativa, aún cuando se excluyen de la muestra a los estudiantes con alguna discapacidad adicional asociada. Un estudio de regresión reveló, asimismo, la importancia de las funciones ejecutivas para los problemas conductuales en los estudiantes con DV. Los resultados demuestran que un amplio rango de dominios de las funciones ejecutivas que son importantes para el desarrollo socioemocional, no están desarrollados suficientemente en muchos estudiantes con DV. Y este desarrollo insuficiente, parece ser particularmente cierto para estudiantes con DV que atienden escuelas especiales.

Por último, De Verdier (2016) reporta un estudio longitudinal que examina el bienestar psicosocial y relaciones sociales en la escuela, de seis estudiantes con DV en el sistema de educación inclusivo sueco. Los resultados revelaron muchos desafíos relacionados con la inclusión social, con una mayoría de familias con posturas críticas respecto de la situación social en las escuelas. Durante los primeros años de escolarización, se pudo apreciar una serie de intervenciones educativas para facilitar la inclusión de los estudiantes con DV. Al crecer los estudiantes, sin embargo, las oportunidades de padres y profesores de incidir en las dinámicas de grupo y crear espacios sociales organizados, disminuyeron dramáticamente. Y aún cuando el bienestar psicosocial reportado por el SDQ no difiere entre niños con DV y sin esta condición, las entrevistas en profundidad revelaron que los estudiantes con DV estaban estresados con la escuela, con problemas con sus compañeros videntes y con sentimientos de soledad.

En síntesis, parece claro que no hay evidencia concluyente respecto a los resultados de la educación segregada versus inclusiva en el caso de estudiantes ciegos y sordos. El presente estudio pretende aportar alguna evidencia en esta dirección, mostrando el caso de 
preescolares sordos y ciegos que atienden ambos tipos de educación. Específicamente buscamos responder las siguientes preguntas: (1) ¿Cómo es el rendimiento de estudiantes con DV y DA en contextos segregados versus inclusivos en rendimiento cognitivo, lingüístico, matemático y emocional? (2) ¿'Se observan diferencias en subgrupos dentro de cada categoría de discapacidad?

\section{Método}

Para responder las preguntas de investigación se diseñó un estudio no experimental transversal de tipo correlacional.

\section{Muestra}

La muestra se conforma por 29 niños con discapacidad visual (DV) y 22 niños con discapacidad auditiva (DA). En el caso de los niños con DV, el promedio de edad de aquellos pertenecientes a escuelas regulares es de 80,16 meses y el promedio de edad de los niños pertenecientes a escuelas especiales es de 86,03 meses. En el Cuadro 1 se presentan los datos relativos al sexo de los niños y al grado de discapacidad.

\section{Cuadro 1}

Descripción de la muestra de niños con $\mathrm{DV}$

\begin{tabular}{lcccc}
\hline & \multicolumn{2}{c}{ ESCUELAS REGULARES } & \multicolumn{2}{c}{ ESCUELAS ESPECIALES } \\
\cline { 2 - 5 } & Hombres & Mujeres & Hombres & Mujeres \\
\hline Baja visión & 2 & 4 & 4 & 3 \\
Ceguera total & 2 & 2 & 7 & 5 \\
\hline
\end{tabular}

Respecto de los niños con DA, el promedio de edad de aquellos pertenecientes a escuelas regulares es de 101,61 meses y el promedio de edad de los niños pertenecientes a escuelas especiales es de 95,79 meses. En el Cuadro 2 se presentan los datos relativos al sexo de los niños y al grado de discapacidad.

\section{Cuadro 2}

Descripción de la muestra de niños con $\mathrm{D} A$

\begin{tabular}{lcccc}
\hline & \multicolumn{2}{c}{ ESCUELAS REGULARES } & \multicolumn{2}{c}{ ESCUELAS ESPECIALES } \\
\cline { 2 - 5 } & Hombres & Mujeres & Hombres & Mujeres \\
\hline Comunicación oral & 2 & 2 & 0 & 0 \\
Lengua de señas & 3 & 1 & 11 & 3 \\
\hline
\end{tabular}

\section{Procedimiento}

Se evaluó el desarrollo de las habilidades cognitivas, los precursores de la lectura, las habilidades matemáticas iniciales y la presencia de problemas emocionales y conductuales en los estudiantes cuando se encontraban finalizando el segundo año de transición de la educación inicial. Las evaluaciones fueron realizadas por profesionales previamente capacitados. Se desarrollaron en los centros educativos o en las casas de los participantes, en dos sesiones individuales de alrededor de 45 minutos. Los niños fueron invitados a participar a través de sus escuelas o sus familias, y solo se incluyeron en el estudio, niños que hubieran sido autorizados por sus padres mediante consentimiento informado y que hubieran demostrado su interés voluntario en participar a través de un asentimiento informado. Se siguieron todos los lineamientos propuestos por el comité de ética de Ciencias Sociales y Humanidades de la Pontificia Universidad Católica de Chile. 


\section{Instrumentos}

Los instrumentos utilizados fueron los siguientes:

- Subtest de Analogías de la prueba WISC-V (Rosas y Pizarro, 2017). Esta prueba evalúa el razonamiento lógico abstracto y el razonamiento verbal ( $\alpha$ de Cronbach $=0.89$ )

- Subtest Gato-Perro de la batería de evaluación de las Funciones Ejecutivas Yellow Red (Rosas et al., 2020). Esta prueba se utilizó para evaluar el desarrollo general de las funciones ejecutivas $(\alpha$ de Cronbach $=0.83)$. Esta prueba fue aplicada solo a los estudiantes con DA.

- Subtest de Retención de Dígitos de la prueba WISC-V. Esta prueba evalúa la memoria auditiva de corto plazo y la memoria de trabajo entre otras habilidades cognitivas $(\alpha$ de Cronbach $=0.89$ ). Se utilizó para niños con DV y se realizó una adaptación lingüística a los niños usuarios de LS.

- Evaluación de Conciencia Fonológica PEFCO (Varela et al., 2015). Se utilizó una adaptación de la prueba a niños con DV ( $\alpha$ de Cronbach $=0.89$ ).

- Paradigma experimental de evaluación del conocimiento de las letras. Se usó la propuesta de Caravolas y su equipo (2013). Esta prueba fue aplicada solo a los estudiantes con DA.

- Prueba de Velocidad de Denominación de Denckla y Rudel. Se utilizó el procedimiento estándar propuesto por Denckla y Rudel (1976). Esta prueba fue aplicada solo a los estudiantes con DA.

- Subtest de Vocabulario de la prueba WISC-V (Rosas y Pizarro, 2017). Se evaluó la profundidad del vocabulario $(\alpha$ de Cronbach $=0.70$ ). Se aplicó la versión tradicional a estudiantes con DV y una adaptación a estudiantes usuarios de LS.

- Test de amplitud de Vocabulario. La amplitud del vocabulario se evaluó con una adaptación de TEVI-R. Se aplicó solo a estudiantes con DA.

- Subtest de Comprensión Oral de las pruebas de Aprovechamiento de la Batería Woodcock-Muñoz (Muñoz-Sandoval, Woodcock, McGrew, y Mather, 2005). Esta prueba fue aplicada a los estudiantes con DV en su versión tradicional ( $\alpha$ de Cronbach $=0.85)$, y fue adaptada lingüísticamente en LS.

- Batería de Evaluación MARKO-D versión chilena. Evaluación de precursores de las matemáticas (Confiabilidad personal=.91). Se adaptó el instrumento tanto para niños con DV como DA.:

- Inventario de Conducta Infantil Child Behaviour Checklist (Achenbach y Rescorla, 2001; Achenbach y Ruffle, 2000). Esta prueba evalúa la presencia de problemas emocionales y conductuales $(\alpha$ de Cronbach $=0.74)$. Este instrumento fue respondido por los padres y apoderados de los niños con DA y DV.

\section{Análisis de datos}

Para analizar las diferencias existentes entre los niños que se educan en escuelas regulares y especiales respecto de las habilidades cognitivas, habilidades precursoras de la lectura, habilidades matemáticas iniciales y problemas emocionales se realizó una comparación de 
medias y un análisis de varianza. Para todos los análisis se utilizó el paquete estadístico SPSS versión 26.

\section{Resultados}

Los resultados se presentan en función de los distintos grupos de niños evaluados: se considera la evaluación de ciegos y sordos por separado, y dentro de cada grupo se presentan tanto los resultados generales comparados por tipo de establecimiento educativo (regular o especial) como por grupo dentro de cada tipo de establecimiento. En el caso de la DV se distingue entre niños ciegos y con baja visión, y en el caso de los niños sordos se distingue entre aquellos que se comunican por medio de lengua de señas chilena y los que se comunican oralmente.

\subsection{Discapacidad visual}

Tal como se puede ver en la Figura 1 y en el Cuadro 3, el desempeño del grupo completo de niños ciegos y con baja visión respecto de las habilidades cognitivas, las habilidades precursoras de la lectura y las habilidades matemáticas iniciales es mejor en el caso de los estudiantes que asisten a educación regular. Sin embargo, solo existen diferencias significativas en el caso de las habilidades matemáticas iniciales $(\mathrm{F}=5.505, \mathrm{p}=.027)$. Por otra parte, en el caso de los problemas emocionales, los estudiantes integrados tienen más problemas que los estudiantes de escuelas especiales. Estas diferencias son estadísticamente significativas $(\mathrm{F}=4.598, \mathrm{p}=.041)$.

\section{Figura 1}

Promedios obtenidos por los niños con discapacidad visual en habilidades cognitivas, precursores de la lectura, matemática inicial y problemas emocionales

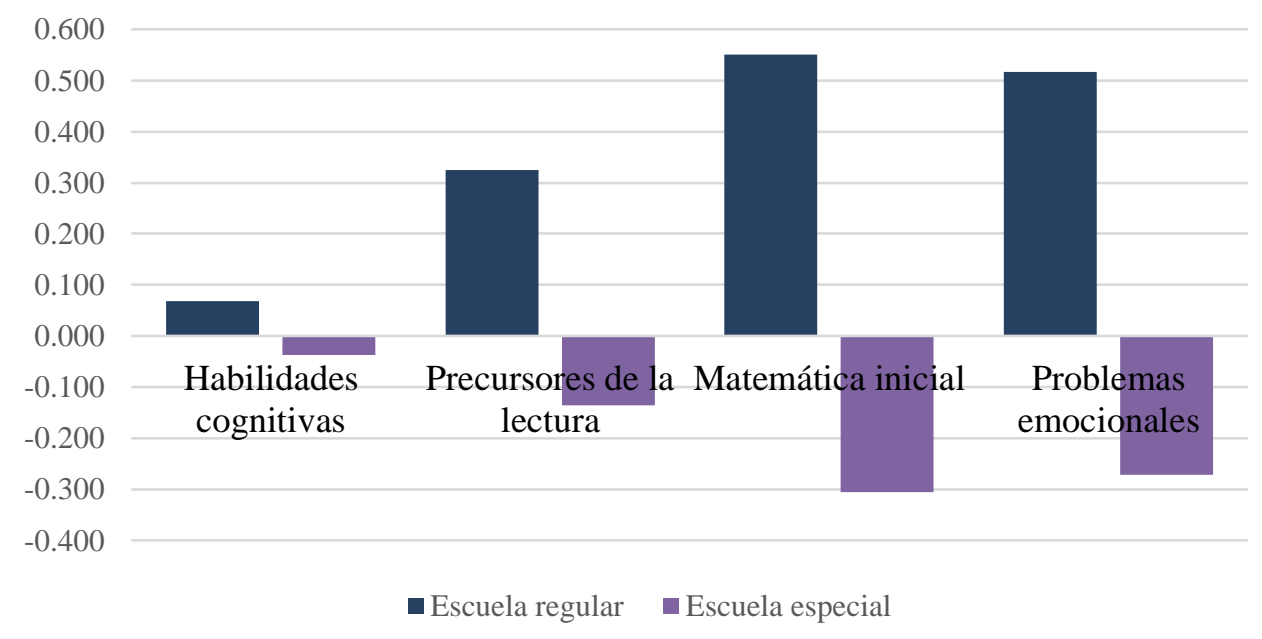




\section{Cuadro 3}

Medias, desviaciones estándar y resultados de ANOVA estudiantes con discapacidad visual

\begin{tabular}{|c|c|c|c|c|c|c|c|}
\hline & \multicolumn{2}{|c|}{ E. REGULAR } & \multicolumn{2}{|c|}{ E. ESPECIAL } & \multirow{2}{*}{$\mathbf{F}$} & \multirow{2}{*}{$\mathbf{P}$} & \multirow{2}{*}{$\eta^{2}$} \\
\hline & $\mathbf{M}$ & DS & $\mathbf{M}$ & DS & & & \\
\hline Habilidades cognitivas & 0,067 & 1,176 & $-0,037$ & 0,923 & 0,068 & 0,796 & 0,003 \\
\hline Precursores de la lectura & 0,324 & 1,035 & $-0,136$ & 0,981 & 1,202 & 0,283 & 0,046 \\
\hline Matemática inicial & 0,551 & 1,001 &,- 0306 & 0,883 & 5,505 & 0,027 & 0,175 \\
\hline Problemas emocionales & 0,517 & 1,115 & $-0,272$ & 0,841 & 4,598 & 0,041 & 0,146 \\
\hline
\end{tabular}

\subsubsection{Estudiantes con baja visión}

Al analizar el comportamiento del grupo de los estudiantes con diagnóstico de baja visión, podemos ver que se mantiene una tendencia favorable en el desempeño de los estudiantes integrados en escuelas regulares respecto de las habilidades cognitivas, los precursores de la lectura y las habilidades matemáticas iniciales, pero sin diferencias significativas. En el caso de los problemas emocionales, se mantiene también la tendencia anterior, existiendo una menor presencia de problemas en el grupo de niños que asisten a escuelas especiales. En este caso las diferencias tampoco son significativas. Esto se puede observar tanto en la Figura 2 como en el Cuadro 4.

\section{Figura 2}

Promedios obtenidos por los niños con baja visión en habilidades cognitivas, precursores de la lectura, matemática inicial y problemas emocionales

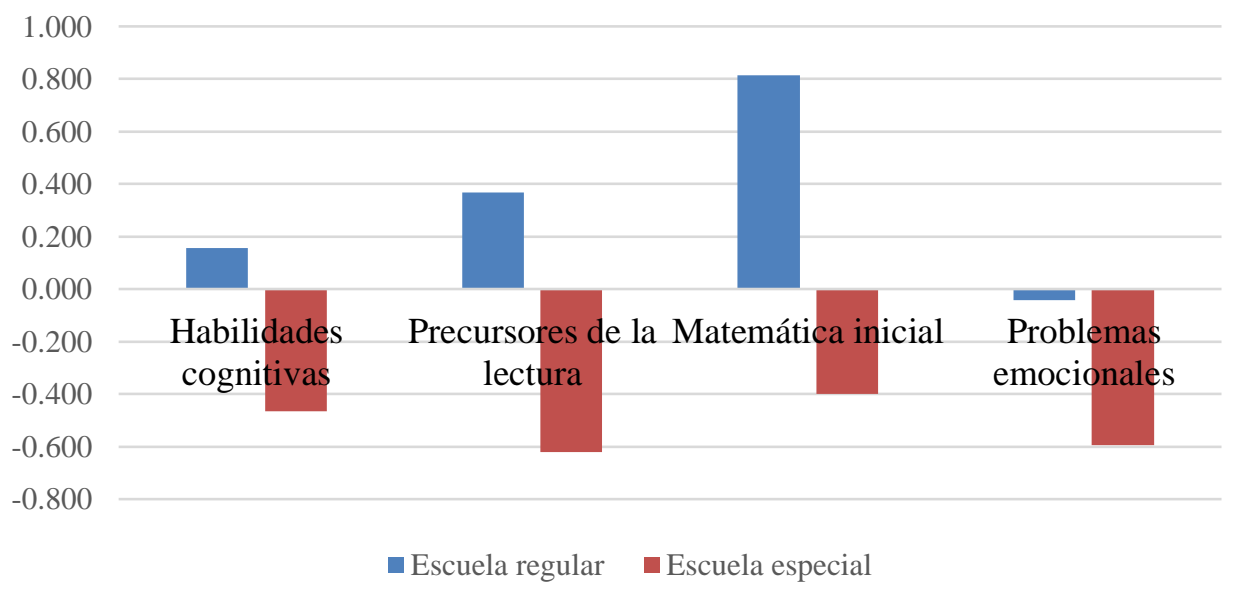

\section{Cuadro 4}

Medias, desviaciones estándar y resultados de ANOVA estudiantes con baja visión

\begin{tabular}{|c|c|c|c|c|c|c|c|}
\hline & \multicolumn{2}{|c|}{ E. REGULAR } & \multicolumn{2}{|c|}{ E. ESPECIAL } & \multirow{2}{*}{$\mathbf{F}$} & \multirow{2}{*}{$\mathbf{P}$} & \multirow{2}{*}{$\eta^{2}$} \\
\hline & M & DS & $\mathbf{M}$ & DS & & & \\
\hline Habilidades cognitivas & 0,156 & 0,967 & $\begin{array}{c}- \\
0,0467\end{array}$ & 1,088 & 1,173 & 0,302 & 0,096 \\
\hline Precursores de la lectura & 0,369 & 0,518 & $-0,620$ & 1,261 & 2,686 & 0,132 & 0,212 \\
\hline Matemática inicial & 0,814 & 0,763 & $-0,399$ & 1,214 & 4,454 & 0,059 & 0,288 \\
\hline Problemas emocionales & $\begin{array}{c}- \\
0,044\end{array}$ & 0,676 & $-0,594$ & 0,812 & 1,724 & 0,216 & 0,135 \\
\hline
\end{tabular}

\subsubsection{Estudiantes con ceguera total}

Al analizar el desempeño de los estudiantes con ceguera total, tanto en la Figura 3 como en el Cuadro 5 podemos observar que la diferencia favorable a los estudiantes integrados 
en escuelas regulares se mantiene en el caso de los precursores de la lectura y de las habilidades matemáticas iniciales, sin que estas lleguen a ser estadísticamente significativas. También se mantiene la tendencia relativa a la presencia de problemas emocionales, donde los estudiantes de escuelas especiales presentan una menor presencia de este tipo de problemas, existiendo una diferencia estadísticamente significativa $(\mathrm{F}=7.380, \mathrm{p}=.017)$. Sin embargo, la tendencia cambia de dirección en el caso del desarrollo de las habilidades cognitivas, donde los estudiantes con ceguera total pertenecientes a escuelas especiales presentan un mayor desarrollo que los estudiantes de las escuelas regulares, aunque las diferencias no son estadísticamente significativas.

\section{Figura 3}

Promedios obtenidos por los niños con ceguera total en habilidades cognitivas, precursores de la lectura, matemática inicial y problemas emocionales

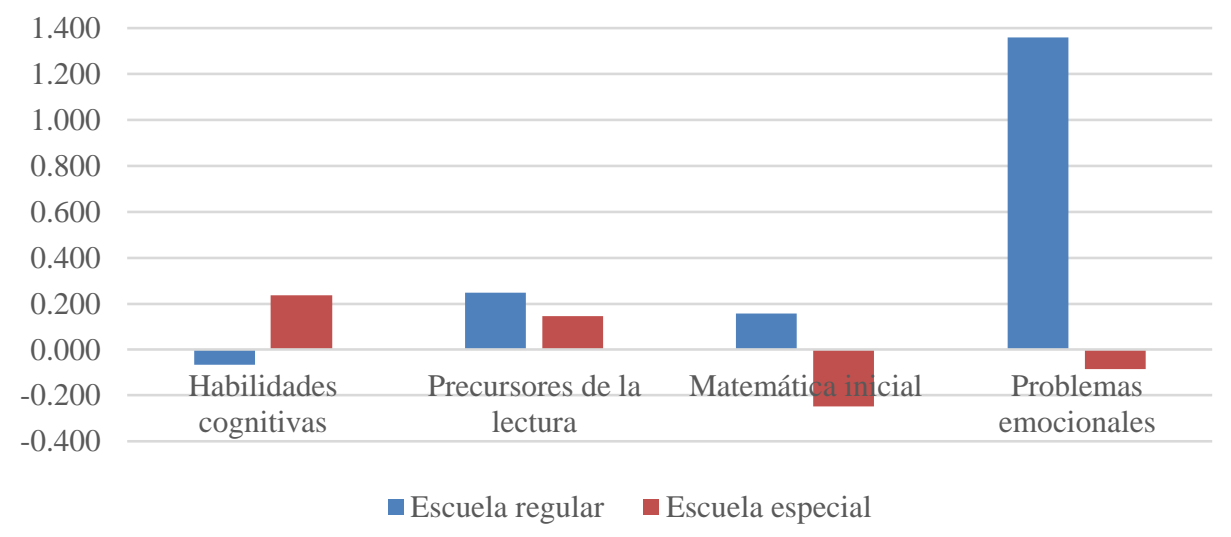

\section{Cuadro 5}

Medias, desviaciones estándar y resultados de ANOVA estudiantes con baja visión

\begin{tabular}{|c|c|c|c|c|c|c|c|}
\hline & \multicolumn{2}{|c|}{ E. REGULAR } & \multicolumn{2}{|c|}{ E. ESPECIAL } & \multirow{2}{*}{$\mathbf{F}$} & \multirow{2}{*}{$\mathbf{P}$} & \multirow{2}{*}{$\eta^{2}$} \\
\hline & $\mathbf{M}$ & DS & $\mathbf{M}$ & DS & & & \\
\hline Habilidades cognitivas & $-0,066$ & 1,600 & 0,236 & 0,726 & 0,367 & 0,614 & 0,020 \\
\hline Precursores de la lectura & 0,248 & 1,789 & 0,146 & 0,686 & 0,029 & 0,868 & 0,002 \\
\hline Matemática inicial & 0,155 & 1,299 & $-0,247$ & 0,658 & 0,656 & 0,433 & 0,048 \\
\hline Problemas emocionales & 1,357 & 1,182 & $-0,084$ & 0,833 & 7,380 & 0,017 & 0,345 \\
\hline
\end{tabular}

\subsection{Discapacidad auditiva}

En el caso de los estudiantes sordos, los análisis se realizarán comparando tres grupos de estudiantes: estudiantes sordos que se comunican oralmente integrados en escuelas regulares, estudiantes que se comunican por lengua de señas chilena (LSch) integrados en escuelas regulares y estudiantes que se comunican por LSch que estudian en escuelas especiales. No se observaron casos de niños sordos que se comunican oralmente en escuelas especiales.

La Figura 4 y el Cuadro 6 muestran los resultados del desempeño de los estudiantes pertenecientes a los distintos grupos. Los estudiantes sordos de escuelas especiales presentan mejor desempeño respecto de las habilidades cognitivas, los precursores de la lectura y las habilidades matemáticas iniciales. Los estudiantes sordos que se comunican de manera oral y que se encuentran integrados en escuelas regulares presentan un desempeño medio en dichas habilidades y, los estudiantes sordos que se comunican con LSch integrados en escuelas regulares son los que presentan un desempeño más 
descendido respecto de las habilidades cognitivas, las habilidades precursoras de la lectura y las habilidades matemáticas iniciales. Finalmente, respecto de la presencia de problemas emocionales, los estudiantes que presentan menor cantidad de problemas son los que se comunican con LSch y se encuentran estudiando en escuelas especiales. Luego, entre los estudiantes que se encuentran integrados en escuelas regulares, son los niños que se comunican oralmente aquellos que manifiestan mayor presencia de problemas emocionales. Sin embargo, ninguna de las diferencias observadas es estadísticamente significativa, por lo que estos hallazgos deben ser interpretados con cautela.

\section{Figura 4}

Promedios obtenidos por los niños con discapacidad auditiva en habilidades cognitivas, precursores de la lectura, matemática inicial y problemas emocionales

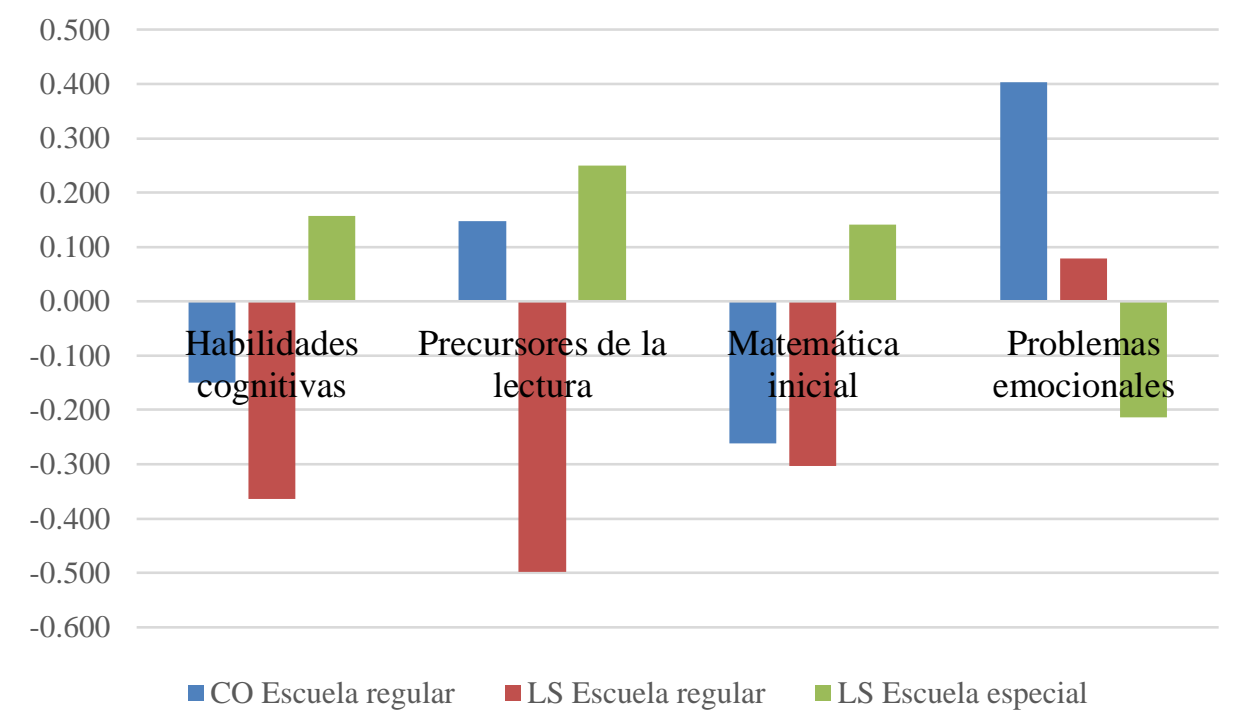

\section{Cuadro 6}

Medias, desviaciones estándar y resultados de ANOVA estudiantes con discapacidad auditiva

\begin{tabular}{|c|c|c|c|c|c|c|c|c|c|}
\hline & \multicolumn{4}{|c|}{ E. REGULAR } & \multirow{2}{*}{\multicolumn{2}{|c|}{$\begin{array}{c}\text { E. ESPECIAL } \\
\text { Lengua de señas }\end{array}$}} & \multirow{3}{*}{$\mathbf{F}$} & \multirow{3}{*}{$\mathbf{P}$} & \multirow{3}{*}{$\eta^{2}$} \\
\hline & \multicolumn{2}{|c|}{ Comunicación oral } & \multicolumn{2}{|c|}{ Lengua de señas } & & & & & \\
\hline & $\mathbf{M}$ & DS & $\mathbf{M}$ & DS & $\mathbf{M}$ & DS & & & \\
\hline 1 & $-0,149$ & 0,722 & $-0,363$ & 0,699 & 0,158 & 0,156 & 0,444 & 0,648 & 0,047 \\
\hline 2 & 0,147 & 1,154 & $-0,497$ & 1,203 & 0,250 & 0,755 & 0,951 & 0,407 & 0,106 \\
\hline 3 & $-0,262$ & 0,844 & $-0,303$ & 0,860 & 0,141 & 1,103 & 0,332 & 0,722 & 0,042 \\
\hline 4 & 0,403 & 1,518 & 0,079 & 0,727 & $-0,214$ & 0,895 & 0,510 & 0,611 & 0,068 \\
\hline
\end{tabular}

Nota. 1: Habilidades cognitivas, 2: Precursores de la lectura, 3: Matemática inicial, 4: Problemas emocionales

\section{Discusión}

Una primera cuestión importante que es preciso destacar de este estudio, es la coincidencia de nuestros resultados en general, con la conocida variabilidad de las condiciones de discapacidad visual y auditiva, lo que hace muy difícil hablar de resultados generalizables para estudiantes de estas dos condiciones. Por el lado de los ciegos, el rango amplio que va de la baja visión a la ceguera total. Por el lado de los sordos, el rango aún más amplio 
de los niños con baja audición, con manejo de lengua de señas vs oralizados, implantados y no, y una larga lista de etcéteras (Archbold, 2015).

Una segunda cuestión importante a destacar, es que ambas condiciones no son comparables, por lo que tanto los resultados como la discusión de estos se hacen exprofeso por separado. Pero siempre teniendo en la mira, que la inclusión de estas dos poblaciones en la educación regular no es igualmente sustentable, como lo muestran claramente los resultados.

Un primer asunto a discutir en el caso de los niños con DV es su rendimiento diferencial en pruebas cognitivas y socioemocionales, según atienden a contextos inclusivos o segregados. Al tomarlos como grupo completo, sin distinción de ceguera o baja visión, se puede afirmar de manera clara que los estudiantes evaluados que asisten a una escuela inclusiva tienen mejor rendimiento en matemáticas iniciales que sus pares de escuelas especiales segregadas. Pero estos últimos tienen una clara ventaja en ajuste socioemocional. No tenemos ninguna referencia para discutir el primer punto, pero el segundo es consistente con los hallazgos de De Verdier (2016) y Heyl y Hintermair (2015), quienes reportan problemas en general en el desarrollo de funciones ejecutivas relacionadas con el desarrollo socioemocional, y sentimientos de soledad y malestar psicosocial de niños integrados, respectivamente. En la misma línea, Heyl y Hintermair, (2015) relacionan funciones ejecutivas con problemas conductuales, siendo particularmente cierto para niños de escuelas especiales. Esto no concuerda con nuestros resultados, pues los niños ciegos pertenecientes a nuestra muestra presentan menos problemas emocionales cuando se encuentran en contextos segregados. Por lo tanto, los resultados de la presente investigación sugieren que es preciso profundizar respecto de si contextos segregados para niños con DV en general, no podrían ser considerados como un factor protector para su desarrollo socioemocional. Esto podría tener implicancias para promover la interacción con pares de niños con DV, incluso en contextos segregados.

En lo que respecta a nuestra investigación, los estudiantes ciegos y con baja visión, en general presentan mejor desarrollo de habilidades cognitivas e instruccionales en la escuela regular, pero las diferencias no son estadísticamente significativas, por lo que es preciso tomar estos resultados con cautela.

Respecto de los estudiantes con DA que participaron de nuestro estudio, se analizó la diferencia por tipo de educación (especial o inclusión en escuela regular) y no por grado de pérdida auditivat. Sin embargo, al comparar a los niños oralizados que en su mayoría eran implantados, los sordos de escuela especial tuvieron mejor rendimiento en todas las medidas, aunque de manera no significativa. Esto contrasta de alguna forma con lo expuesto por Mitchell y Karchmer, (2012), quienes plantean que los sordos profundos presentan menor rendimiento que los moderados, pero sin mencionar a qué tipo de escuela asistían los niños de ese estudio.

La divergencia con respecto a los resultados obtenidos por niños sordos en los diversos contextos (escuelas especiales o regulares) junto con la baja significancia estadística de los resultados de esta investigación asociados a esto, invita a retomar la idea de Stinson y

\footnotetext{
${ }^{4}$ La pérdida auditiva no siempre es total, sino que podrá tener distintos niveles de severidad. En esta línea, se identifican cuatro grados de pérdida auditiva: ligera/leve, moderada, intensa/severa o profunda (Organización Mundial de la Salud, 2016).
} 
Kluwin (2012), quienes proponen que juzgar la efectividad de diferentes sistemas educativos para los estudiantes sordos, es "difícil, si no imposible, debido a las diferencias de los niños que asisten a estos programas” (p. 51). En esta línea, antes de realizar cualquier tipo de generalización respecto del sistema de educación regular por sobre el de educación especial o viceversa, es relevante comprender de manera más exhaustiva la incidencia del resto de los factores en dichos resultados.

Un factor importante que podría explicar por qué los sordos se desempeñan mejor en escuelas especiales, es la formación de los profesores, fundamentalmente debido a su conocimiento de didácticas específicas y del dominio de la lengua de señas. No hay un esfuerzo del sistema "inclusivo" por adaptar las clases a las necesidades de estos estudiantes y muy rara vez los profesores regulares dominan la lengua de señas. Esto coincide con los planteado Marschark y otros (2002).

Este resultado se ve reforzado por el hecho que en nuestro estudio los sordos con menores problemas emocionales son los que se encuentran en escuelas especiales. Esto puede estar relacionado con las posibilidades de comunicación con sus pares, lo que coincide con lo planteado por Stinson y Kluwin (2012).

No es lo mismo ir a una escuela regular que a una escuela especial segregada, no obstante, quizás aún carecemos de evidencia que nos permita concluir de manera certera dónde radican dichas diferencias o qué tipo de educación es más indicada en cada caso. Lo que sí se puede concluir, es que los efectos de la inclusión no son siempre positivos. Una escuela inclusiva debe ser pensada y articulada por todos los miembros de la comunidad educativa y no solo por aquellos que se relacionan con el área discapacidad de una u otra manera (educadores diferenciales y estudiantes con discapacidad).

A nivel general, creemos que los resultados reflejan las implicancias de un sistema que no es realmente inclusivo, sino de uno basado en el paradigma de la integración. En las áreas donde no es necesaria una estrategia que aborde a todo el curso o a la escuela en su globalidad no hay problemas (como en las áreas instruccionales en el caso de los ciegos), pero cuando los esfuerzos personales e institucionales no son suficientes (por más que un niño sordo quiera comprender lo que dice su profesor, no va a poder hacerlo si no cuenta con los apoyos y adecuaciones necesarias), la inclusión no asegura mejores efectos educativos que la escuela especial.

Es importante avanzar en la identificación y definición de cuáles son las condiciones reales de la inclusión educativa de estudiantes con DV y DA en nuestro país, para proveer de las condiciones que permitan un adecuado desarrollo cognitivo y emocional de los estudiantes. No basta con tener al niño sordo en la sala, y que pueda contar con un intérprete. Los profesores deben aprender cómo enseñar a este estudiante, y además la escuela debe establecer estrategias para una positiva integración social.

De acuerdo a lo que plantean Antia y otros (2011), podría lograrse un buen desarrollo emocional si se consideran apoyos profesionales en diversas áreas, lo cual exige, además, un esfuerzo intencionado de padres y educadores que se traduzca en un mayor número de interacciones positivas de estos con sus pares (Wolters, 2014).

En esta línea, tanto en el caso de la DV como en la DA, se torna necesario contar con profesionales especializados actualizados en base a la evidencia científica, de modo de avanzar en la elaboración estrategias de apoyo e intervenciones personales y contextuales apropiadas para cada niño según su NEE, características personales y contexto. 


\section{Agradecimientos}

Esta investigación fue financiada por ANID PIA CIE 160007 y desarrollada por el Centro de Justicia Educacional de la Universidad Católica de Chile.

\section{Referencias}

Achenbach, T. M. y Rescorla, L. A. (2001). Manual for the ASEBA school-age forms \& profiles. University of Vermont, Research Center for Children, Youth, \& Families.

Achenbach, T. M. y Ruffle, T. M. (2000). The child behavior checklist and related forms for assessing behavioral/emotional problems and competencies. Pediatrics in Review, 21(8), 265271. https://doi.org/10.1542/pir.21-8-265

Alabdulkader, B. y Leat, S. J. (2010). Lectura en niños con baja visión. Journal of Optometry, 3(2), 68-73. https://doi.org/10.1016/S1888-4296(10)70010-8

Antia, S. D., Jones, P., Luckner, J., Kreimeyer, K. H. y Reed, S. (2011). Social outcomes of students who are deaf and hard of hearing in general education classrooms. Exceptional Children, 77(4), 489-504. https://doi.org/10.1177/001440291107700407

Archbold, S. (2015). Being a deaf student. En VVAA, Educating deaf learners (pp. 23-46). Oxford University Press. https://doi.org/10.1093/acprof:oso/9780190215194.003.0002

Archbold, S., Nikolopoulos, T. P., Lutman, M. E. y O’Donoghue, G. M. (2002). The educational settings of profoundly deaf children with cochlear implants compared with age-matched peers with hearing aids: implications for management. International Journal of Audiology, $41(3), 157-161$.

Biermann, J. y Powell, J. J. W. (2014). Institutionelle dimensionen inklusiver schulbildung herausforderungen der un-behindertenrechtskonvention für Deutschland, Island und Schweden im Vergleich. Zeitschrift Fur Erziehungswissenschaft, 17(4), 679-700. https://doi.org/10.1007/s11618-014-0588-0

Biermann, J. y Powell, J. J. W. (2016). From exclusion and segregation to inclusion? Dis/abilitybased inequalities in the education systems of Germany and Nigeria. En A. Hadjar y C. Gross (Eds.), Education systems and inequalities (pp. 207-230). Policy Press. https://doi.org/10.1332/policypress/9781447326106.003.0011

Caravolas, M., Lervåg, A., Defior, S., Málková, G. S. y Hulme, C. (2013). Different patterns, but equivalent predictors of growth in reading in consistent and inconsistent orthographies. Psychological Science, 24(8), 1398-1407. https://doi.org/10.1177/0956797612473122

Celeste, M. y Grum, D. K. (2010). Social integration of children with visual impairment: A developmental model. Illö̈̆retim Online, 9(1), 11-22. https://doi.org/10.17051/io.29276

Ching, T. Y. C., Dillon, H., Leigh, G. y Cupples, L. (2018). Learning from the Longitudinal Outcomes of Children with Hearing Impairment (LOCHI) study. International Journal of Audiology, 57, S105-S111. https://doi.org/10.1080/14992027.2017.1385865

Contreras, D., Brante, M., Espinoza, S. y Zuñiga, I. (2020). The effect of the integration of students with special educational needs: Evidence from Chile. International Journal of Educational Development, 74, 102163. https://doi.org/10.1016/j.ijedudev.2020.102163

Crowe, K. (2018). Deaf and hard-of-hearing multilingual learners. Oxford University Press. https://doi.org/10.1093/oso/9780190880545.003.0003 
Cupples, L., Ching, T. Y. C., Button, L., Seeto, M., Zhang, V., Whitfield, J., Marnane, V. (2018). Spoken language and everyday functioning in 5-year-old children using hearing aids or cochlear implants. https://doi.org/10.1080/14992027.2017.1370140

De Raeve, L., Baerts, J., Colleye, E. y Croux, E. (2012). Changing schools for the deaf: Updating the educational setting for our deaf children in the 21 st century, a big challenge. Deafness and Education International, 14(1), 48-59.

https://doi.org/10.1179/1557069X11Y.0000000012

De Verdier, K. (2016). Inclusion in and out of the classroom: A longitudinal study of students with visual impairments in inclusive education. British Journal of Visual Impairment, 34(2), 132142. https://doi.org/10.1177/0264619615625428

Denckla, M. B. y Rudel, R. (1976). Rapid “automatized” naming (RAN): Dyslexia differentiated from other learning disabilities. Neuropsychologia, 14, 471-479.

https://doi.org/10.1016/0028-3932(76)90075-0

Dyck, M. J., Farrugia, C., Shochet, I. M. y Holmes-Brown, M. (2004). Emotion recognition/understanding ability in hearing or vision-impaired children: Do sounds, sights, or words make the difference? Journal of Child Psychology and Psychiatry and Allied Disciplines, 45(4), 789-800. https://doi.org/10.1111/j.1469-7610.2004.00272.x

Farrell, M. (2010). Debating special education. Routledge. https://doi.org/10.4324/9780203852453

Goldberg, L. R. y Richburg, C. M. (2004). Minimal hearing impairment: Major myths with more Than Minimal Implications. Communication Disorders Quarterly, 25(3), 152-160. https://doi.org/10.1177/15257401040250030601

Heyl, V. y Hintermair, M. (2015). Executive function and behavioral problems in students with visual impairments at mainstream and special schools. Journal of Visual Impairment and Blindness, 109(4), 251-263. https://doi.org/10.1177/0145482x1510900402

Hintermair, M. (2013). The contribution of perceived parental support to the career self-efficacy of deaf, hard-of-hearing, and hearing adolescents. Journal of Deaf Studies and Deaf Education, 18(3), 344-359. https://doi.org/10.1093/deafed/entoo3

Kauffman, J. y Hallahan, D. (2011). Handbook of special education. En VVAA, Handbook of Special Education. Routledge.

Knoors, H. y Marschark, M. (2014). Learning and context. En VVAAA, Teaching Deaf Learners (pp. 216-233). Oxford University Press.

https://doi.org/10.1093/acprof:oso/9780199792023.003.0011

Lindsay, G. (2007). Educational psychology and the effectiveness of inclusive education/mainstreaming. British Journal of Educational Psychology, 77(1), 7-24. https://doi.org/10.1348/000709906X156881

Marschark, M., Lang, H. y Albertini, J. (2002). Educating deaf students: From research to practice. Oxford University Press.

Marschark, M., Sapere, P., Convertino, C. y Pelz, J. (2008). Learning via direct and mediated instruction by deaf students. Journal of Deaf Studies and Deaf Education, 13(4), 546-561. https://doi.org/10.1093/deafed/enno14

Metatla, O. (2017, julio). Uncovering challenges and opportunities of including children with visual impairments in mainstream schools. Comunicación presentada en el HCI 201 7: Digital Make Believe - Proceedings of the 31 st International BCS Human Computer Interaction Conference. https://doi.org/10.14236/ewic/HCI2017.102 
Mitchell, R. E. y Karchmer, M. A. (2012). The Oxford handbook of deaf studies, language, and education. Oxford. https://doi.org/10.1093/oxfordhb/9780199750986.013.0003

Moeller, M. P., Tomblin, J. B., Yoshinaga-Itano, C., Connor, C. M. D. y Jerger, S. (2007). Current state of knowledge: Language and literacy of children with hearing impairment. Ear and Hearing, 28(6), 740-753. https://doi.org/10.1097/AUD.0bo13e318157fo7f

Moog, J. S., Geers, A. E., Gustus, C. H. y Brenner, C. A. (2011). Psychosocial adjustment in adolescents who have used cochlear implants since preschool. Ear and Hearing, 32. https://doi.org/10.1097/aud.obo13e3182014c76

Morris, M. y Smith, P. (2008). Educational provision for blind and partially sighted children and young people in England. Routledge.

Muñoz-Sandoval, A. F., Woodcock, R. W., McGrew, K. S. y Mather, N. (2005). Batería III Woodcock-Muñoz. Riverside Publishing.

Organización Mundial de la Salud. (2016). Pérdida de audición en la niñez iQué hacer para actuar de inmediato! OMS.

Rosas, R., Espinoza, V. y Garolera, M. (2020). Evidencia intercultural de un test basado en Tablet para medir las funciones ejecutivas de niños entre 6 y 10 años: Resultados preliminares. CEDETi UC.

Rosas, R., Staig, J., Lazcano, G., Palacios, R., Espinoza, V., Aro, M. y Imbernón, C. (2019). ¿Qué podemos aprender de los sistemas educativos de Chile, España y Finlandia en el marco de la declaración de Salamanca? Revista Latinoamericana de Educación Inclusiva, 13(2), 57-74. https://doi.org/10.4067/s0718-73782019000200057

Stinson, M. S. y Kluwin, T. N. (2012). Educational consequences of alternative school placements. En VVAA., The Oxford handbook of deaf studies, language, and education (pp. 1-30). https://doi.org/10.1093/oxfordhb/9780199750986.013.0005

Varela, V., De Barbieri, Z., Beltrán, J., Godoy, C., Guerra, A. y Sánchez, M. (2015). PECFO. Prueba de evaluación de conciencia fonológica. Ediciones Pontificia Universidad Católica.

Wolters, N., Knoors, H., Cillessen, A. H. N. y Verhoeven, L. (2014). Social adjustment of deaf early adolescents at the start of secondary school: The divergent role of withdrawn behavior in peer status. Exceptional Children, 80(4), 438-453.

https://doi.org/10.1177/0014402914527242

\section{Breve CV de los/as autores/as}

\section{Ricardo Rosas}

Psicólogo de la Pontificia Universidad Católica de Chile y Doctorado en Psicología Cognitiva de la Freie Universität Berlin. Profesor Titular de la Escuela de Psicología de la PUC. Sus áreas de interés son la Inteligencia, la Psicología de las Discapacidades, el Juego y Aprendizaje Implícito y el desarrollo de sistemas instruccionales basados en tecnologías. Es autor de cuatro libros y numerosas publicaciones en revistas internacionales en sus temas de investigación. Actualmente dirige el Centro de Desarrollo de Tecnologías de Inclusión de la PUC, CEDETi-UC que ha recibido dos importantes premios internacionales: WISE Award 2011 por el programa SUEÑALETRAS, diseñado para ayudar en la mediación de la lectura de niños sordos y el Premio Reina Letizia 2018, en la categoría discapacidad y Rehabilitación. ORCID ID: https://orcid.org/0000-00023091-4044. Email: rrosas@uc.cl 


\section{Victoria Espinoza}

Directora de Investigación del Centro de Desarrollo de Tecnologías de Inclusión y colaboradora en la linea de Investigación y Discapacidad del Centro de Justicia Educacional. Profesora de Educación Básica especialista en Dificultades de Aprendizaje e Inclusión Educativa, Magíster en Psicología, mención en Psicología Educacional, Doctora en Psicología de la Pontificia Universidad Católica de Chile. Sus temas de investigación refieren al proceso de adquisición de la lectura, la educación inicial, el desarrollo de las funciones ejecutivas, la inclusión educativa de estudiantes con discapacidad y las brechas de aprendizaje terminadas por el nivel socioeconómico y su impacto en las posteriores trayectorias de aprendizaje. ORCID ID: https://orcid.org/O000-0001-9462-1139 Email: victoriaespinoza@uc.cl

\section{Elisa Hohlberg}

Psicóloga Orchard College. Titulada en Psicología de la Pontificia Universidad Católica de Chile. Sus áreas de interés son la educación inclusiva, competencias socioemocionales, generación y estandarización de instrumentos de evaluación inclusivos, trayectorias del desarrollo cognitivo, socioemocional y lenguaje en estudiantes con y sin discapacidad, neuropsicología, instrumentos de evaluación inclusivos y aplicación del modelo de Rash para la estandarización de instrumentos de evaluación desde una perspectiva del desarrollo. Actualmente se desempeña como psicóloga en el Colegio Orchard y previamente como coordinadora de terreno de la Línea de Inclusión de la Discapacidad del Centro de Justicia Educacional UC. ORCID ID: https://orcid.org/0000-0003-22156789. Email: mehohlberg@uc.cl

\section{Sanndy Infante}

Psicóloga clínica titulada, Pontificia Universidad Católica de Chile. Magíster en Educación, PUC. Asimismo, es Lingüísta y Licenciada en Filosofía de la misma casa de estudios. Posee estudios de postítulo en Pedagogía para la Educación superior (PUC), Neuropsicología a través del ciclo vital (PUC), y Filosofía política, educación e identidades (Universidad de Chile). En la actualidad, se encuentra cursando su segundo magíster en la Escuela de Psicología UC, en Psicología Clínica. Sus áreas de interés son la didáctica clínica, la neuropsicología, la psicología hospitalaria, la bioética y la lingüística aplicada. Se desempeña como académica de la Facultad de Medicina de la Pontificia Universidad Católica de Chile y, al mismo tiempo, como psicóloga clínica en la Red Salud UC-Christus. ORCID ID: https://orcid.org/O000-0002-3515-5446. Email: spinfant@uc.cl 